\title{
Effects of Photo-Biomodulation in Stargardt Disease
}

\author{
Sergio Zaccaria Scalinci' \\ Nicola Valsecchi (iD ${ }^{2}$ \\ Elena Pacella (iD ${ }^{3}$ \\ Edoardo Trovato Battagliola (iD) ${ }^{3}$ \\ 'Ophthalmology Unit, DIMEC \\ (Department of Medical and Surgical \\ Sciences), Department of \\ Ophthalmology, Alma Mater Studiorum \\ University of Bologna, Bologna, Italy; \\ ${ }^{2}$ Ophthalmology Unit, DIMES \\ (Department of Experimental, Diagnostic \\ and Specialty Medicine), Department of \\ Ophthalmology, Alma Mater Studiorum \\ University of Bologna, Bologna, Italy; \\ ${ }^{3}$ Department of Sense Organs, Faculty of \\ Medicine and Dentistry, Sapienza \\ University of Rome, Rome, Italy
}

Correspondence: Nicola Valsecchi Ophthalmology Unit, DIMES (Department of Experimental, Diagnostic and Specialty Medicine), Department of Ophthalmology, Alma Mater Studiorum University of Bologna, Via Pelagio Pelagi 9, Bologna, 40I38, Italy

Tel +393407783914

Email nicola.valsecchi2@studio.unibo.it
Purpose: Stargardt disease (STGD1) is the most common hereditary macular degeneration and currently no treatments have been approved to interrupt the progression of the disease. The aim of this study was to evaluate the efficacy of photo-biomodulation in patients with Stargardt disease 1 year after treatment, considering as primary outcomes improvements in best corrected visual acuity (BCVA), microperimetry, and pattern electroretinography (PERG) amplitude.

Materials and Methods: Ninety eyes of 45 patients with a diagnosis of Stargardt disease stage 1 were prospectively recruited at the University of Bologna. Selected patients were treated with photo-biomodulation therapy for 1 year for 10 minutes a day, twice a day, and 5 days per week for 12 months. A light emitting diode (LED) of $10 \mathrm{~Hz}$ and wavelength $650 \mathrm{~nm}$ was used. BCVA and microperimetry were assessed before treatment and 1 year after photobiomodulation, whereas PERG was assessed at baseline, 1, 3, 6, and 12 months after treatment.

Results: BCVA improved from 0.7 [interquartile range $(\mathrm{IQR})=0.6-0.9)$ ] to $0.4(\mathrm{IQR}=0.2-$ $0.5)$ ] after treatment $(95 \% \mathrm{CI}=-0.3,-0.35 ; p=<0.001)$ and PERG negative wave at $35 \mathrm{~ms}$ (N35)-positive wave at $50 \mathrm{~ms}$ (P50) amplitude improved from 0.9 (IQR=0.74-1.21) to 2.12 (95\% CI=1.11, 1.28; $p<0.001)$. MP-1 Microperimetry improved from 26.5\% (IQR=16.75$40)$ of median preferred retinal loci (PRL) within an area of $2^{\circ}$ of diameter to $38 \%(\mathrm{IQR}=29$ $50.25 ; 95 \% \mathrm{CI}=10.50,14.50 ; p=<0.001)$; on the other hand, PRL within $4^{\circ}$ of diameter raised from $72 \%(\mathrm{IQR}=51.5-80)$ to $80 \%(\mathrm{IQR}=76-87)$ after photo-biomodulation $(95 \% \mathrm{CI}=4.50$, $7.50 ; p<0.001)$. Also, patients with an improvement of more than 1.205 PERG N35-P50 ratio amplitude at 3 months and more than 1.29 at 6 months tended to present an improvement of more than $0.2 \log$ Mar in BCVA at 12 months $(p=0.28, \mathrm{AUC}=0.630 ; p=0.30, \mathrm{AUC}=0.636$ ).

Conclusion: BCVA, PERG, and MP-1 significantly improved 1 year after treatment. Also, improvement of PERG N35-P50 amplitude at 3 and 6 months was correlated to BCVA improvement at 1 year, suggesting that PERG could be useful in predicting visual outcome after photo-biomodulation.

Keywords: Stargardt disease, PERG, microperimetry, LED, photo-biomodulation

\section{Introduction}

Stargardt disease (STGD1) is a common hereditary macular degeneration and it affects patients with a median age of 27 years old, with a prevalence of 1 in 10,000 persons. $^{1,2}$ It is caused by a mutation in the adenosine triphosphate binding cassette transporter 4 (ABCA4) gene in the chromosome $1^{3}$ and it is inherited recessively, even though an autosomal dominant pattern has been described. ${ }^{4,5}$ It is characterized by a progressive and irreversible bilateral loss of central vision, due to an accumulation of fish-shaped lipofuscin deposits, so called "flecks", in the retinal pigment epithelium (RPE) in the paramacular region. Atrophy of the photoreceptors, RPE, and choriocapillaris (CC) are common features of the disease; though the 
posterior pole is commonly affected, the retinal periphery can also be involved. ${ }^{6}$ Fishman ${ }^{7}$ described four stages of the diseases, based on ophthalmoscopic findings, electrophysiological and psychophysiological tests. Currently, no treatments for STGD1 have been approved in clinical practice, even though different strategies have been tested. ${ }^{8}$ Photo-biomodulation is a low-level laser therapy that penetrates into tissues and activates cytochrome $\mathrm{C}$ oxidase inside the mitochondria, upregulating their metabolism and inducing the production of ATP, thus preventing cell apoptosis. ${ }^{9-11}$ Using a pulsed red Light Emitting Diode (LED) of $650 \mathrm{~nm}$, photo-biomodulation can upregulate mitochondrial cytochrome $\mathrm{c}$ oxidase in the retinal tissue, increase adenosine triphosphate (ATP) and promote Melatonin secretion. ${ }^{12}$ Photo-biomodulation has been tested in dry and wet age-related macular degeneration, where it has been shown to improve visual acuity and to decrease the volume of drusen; ${ }^{13-15}$ moreover, photobiomodulation showed clinical improvements in patients with amblyopia and retinitis pigmentosa. ${ }^{16,17}$ A previous study by Scalinci et al, ${ }^{18}$ in 2015 showed that photobiomodulation was effective in improving best corrected visual acuity (BCVA), microperimetry, and pattern electroretinography (PERG) amplitude after 3months of treatment in patients with Stargardt disease. PERG evaluates macular function measuring two main components: a positive wave at $50 \mathrm{~ms}$ (P50) that depends on macular integrity; and a negative wave at $95 \mathrm{~ms}$ that evaluates ganglion cells function. ${ }^{19}$ On the other hand, microperimetry provides a precise evaluation of macular sensitivity and has already been validated as an important outcome measure in Stargardt disease. ${ }^{20}$

The aim of the current study was to evaluate the efficacy of photo-biomodulation in patients with Stargardt disease in Fishman stage 11 year after treatment considering BCVA, microperimetry, and PERG as primary outcomes. To our knowledge, this is the first prospective study that evaluates the effect of photo-biomodulation after 1 year of treatment in Stargardt disease.

\section{Materials and Methods}

\section{Patients Selection}

The study was prospectively conducted at the University of Bologna in the Policlinico Sant'Orsola Hospital and approved by the institutional review board of the University of Bologna. All procedures were performed in accordance with the ethical standards of the institutional review board and with the Declaration of Helsinki and its later amendments or comparable ethical standards. The participants provided informed consent and patient information was anonymized and de-identified prior to analysis.

Forty-five patients with a diagnosis of Stargardt based on genetic analysis disease were recruited. Inclusion criteria were a Stargardt disease stage 1 according to Fishman classification and BCVA better than 1.0 Logmar at the first eye examination.

Excluding criteria were concomitant epilepsy, neurological diseases, and psychiatric comorbidities. The primary outcome of the study was to evaluate if photobiomodulation could improve BCVA, microperimetry, and PERG N35-P50 amplitude 1 year after treatment. As secondary outcomes, we investigated if improvements in BCVA were correlated to increases in PERG amplitude and microperimetry. Also, we investigated if improvements in PERG amplitude at 1, 3, and 6 months could estimate improvement of BCVA at 12 months.

\section{Clinical Evaluation and Treatment Procedure}

All patients underwent a comprehensive eye examination before treatment. Best corrected visual acuity (BCVA) was tested using the Early Treatment Diabetic Retinopathy Scale (ETDRS). Nidek MP-1 Microperimetry (Nidek Technologies, Italy) color fundus photography was used with an automated program and 4-2 threshold, in order to test fixation location. Macular sensitivity was not taken into account for statistical analysis. PERG transient analysis was conducted to test if photo-biomodulation could improve macular function. N35-P50 amplitude was taken into account for statistical analysis.

Optical Coherence Tomography (OCT) and Fluorescein Angiography (FA) were performed only at the baseline evaluation; for this reason, structural data of the retina were not considered in the statistical analysis.

Selected patients were treated with photobiomodulation therapy for 1 year, using Mnemosline glasses from Telea Electronic Engineering. Mnemosline glasses were used for 10 minutes a day, twice a day, 5 days per week for 12 months. LED pulsed light of $10 \mathrm{~Hz}$ and a wavelength of $650 \mathrm{~nm}$ was used in order to gain the best penetrative capacity in the retina without activating cells apoptosis. Patients were asked to fill in a daily questionnaire at home in order to control treatment compliance. Patients underwent a PERG examination at 1 month, 3 
Table I Demographic Data and Median Values of BCVA, MP-I $2^{\circ}$ and MP-I $4^{\circ}$ Before and After Photo-Biomodulation

\begin{tabular}{|l|l|l|l|l|}
\hline & $\mathbf{n}(\%)$ or Mean (SD) & Baseline (Median, IQR) & I Year (Median, IQR) & P-value \\
\hline Age & $20( \pm 3)$ & & & \\
Female & $27(60 \%)$ & $0.7(0.6-0.9)$ & $0.4(0.2-0.5)$ & $<0.001$ \\
BCVA & & $26.5 \%(16.75-40)$ & $38 \%(29-50.25)$ & $<0.001$ \\
MP-I $2^{\circ}$ & & $72 \%(51.5-80)$ & $80 \%(76-87)$ & $<0.001$ \\
MP-I $4^{\circ}$ & & & \\
\hline
\end{tabular}

Abbreviations: SD, standard deviation; IQR, interquartile range; BCVA, Best Corrected Visual Acuity; MP-I, microperimetry.

months, and 6 months in order to assess changes in amplitude. Twelve months after beginning treatment, the patients were revaluated and BCVA, PERG, and microperimetry were assessed.

\section{Statistical Analysis}

For statistical analysis, normality was tested with the Shapiro-Wilk test and non-parametric test. Wilcoxon signed rank test was used to compare BCVA, PERG, and MP-1 microperimetry before and after photo-biomodulation, in order to evaluate if photo-biomodulation could improve visual acuity, fixation location, and the function of retinal ganglion cells after treatment. Spearman's rank correlation was used to test correlations between pre-post BCVA, PERG and MP-1, in order to evaluate if visual acuity improvement was correlated with improvements in PERG and MP-1; also, it was used to evaluate pre-post BCVA and improvements in PERG at 1, 3, and 6 months. Receiving operating characteristic (ROC) curves were conducted to test if improvements of PERG at 1,3, and 6 months were correlated with an improvement in BCVA, with the aim of evaluating if PERG improvement could estimate improvement in visual acuity during the course of the treatment. The cut-off of 0.2 LogMar was determined after study enrollment in order to evaluate the correlation between PERG improvement and BCVA improvement. Cut-off values were identified with best
Youden's Index (Sensitivity + Specificity -1 ) and tested with Chi-square. Median and interquartile range intervals (IQR) were considered for reporting values. $P$-values $<0.05$ were considered as statistically significant. Statistical analysis was performed using IBM Statistical Package for Social Sciences version 26.

\section{Results \\ Primary Outcomes}

In this study, 90 eyes of 45 patients with Stargardt were recruited, 27 females and 18 males, mean age 20 years old $(\mathrm{SD} \pm 3$ ) (see Table 1). No side-effects were reported during treatment. Before treatment, median BCVA was 0.7 (IQR=0.6-0.9); after treatment, BCVA improved significantly, raising to 0.4 (IQR $=0.2-0.5 ; 95 \% \mathrm{CI}=-0.3,-0.35$; $p=<0.001$ ) (see Figure 1).

The PERG N35-P50 ratio amplitude improved significantly, increasing from 0.9 (IQR=0.74-1.21) before treatment to $2.12(\mathrm{IQR}=1$. 97-2.39) 12months after photo-biomodulation (95\% CI=1.11, 1.28; $p<0.001)$. The improvement was greater in the first 3 months, increasing from 0.9 to 2.0 , whereas it increased slowly in the following months, moving from 2.01 at 3 months to 2.12 at 12 months (see Figure 2).

P-1 microperimetry showed a statistically significant improvement in fixation location: $26.5 \%(\mathrm{IQR}=16.75-40)$
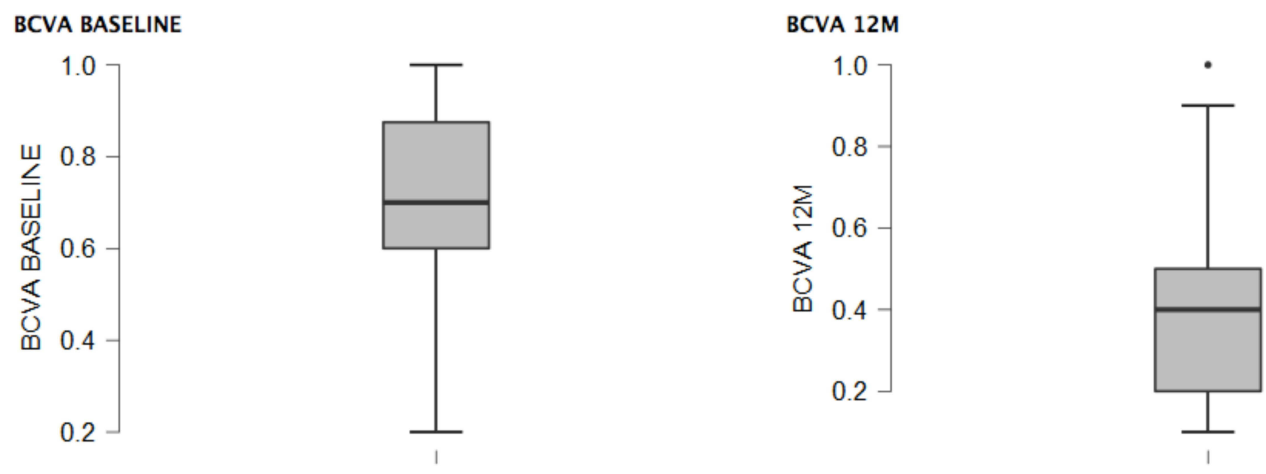

Figure I The box-plot shows BCVA at baseline and BCVA after 12 months of photo-biomodulation. 
PERG N35 - P50 Amplitude

\section{2,5}

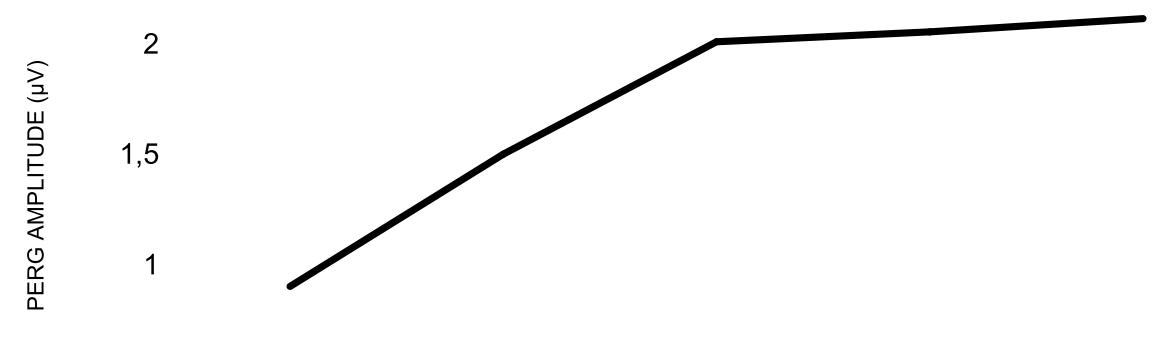

0,5

0

Basal 1 month 3 month 6 month 12 month

TIME AFTER BEGINNING OF TREATMENT (MONTHS)

Figure 2 Median values of PERG amplitude at baseline, I month, 3 months, 6 months, and 12 months after photo-biomodulation.

of median preferred retinal loci (PRL) were within an area of $2^{\circ}$ of diameter, whereas after treatment, PRL increased to $38 \% \quad(\mathrm{IQR}=29-50.25 ; 95 \% \quad \mathrm{CI}=10.50$, 14.50; $p=<0.001)$; on the other hand, PRL within $4^{\circ}$ of the diameter raised from $72 \%(\mathrm{IQR}=51.5-80)$ to $80 \%$ $(\mathrm{IQR}=76-87)$ after photo-biomodulation $(95 \% \mathrm{CI}=4.50$, 7.50; $p<0.001$ ) (see Figure 3).

\section{Secondary Outcomes}

No statistically significant correlations were encountered comparing pre-post BCVA, PERG and MP-1 at 12 months (respectively, $\rho=1.91, p=0.071 ; \rho=0.05 ; \rho=0.060, p=0.574$; $\rho=-0.003, p=0.978)$. On the other hand, BCVA improvement at 12 months was correlated with improvements of PERG N35-P50 ratio at 3 and 6 months (respectively, $\rho=0.214,95 \% \mathrm{CI}=-0.02,0.413, p=0.042 ; \rho=0.236,95 \%$ $\mathrm{CI}=0.028,0.423, p=0.025)$, whereas it did not correlate with PERG improvement at 1 month $(\rho=0.103, p=0.332)$. ROC curve showed that patients with an improvement of more than 1.205 PERG amplitude at 3 months and more than 1.29 at 6 months tended to present an improvement of more than $0.2 \log$ Mar in BCVA at 12 months $(p=0.28$, 95\% $\quad \mathrm{CI}=0.514, \quad 0.747, \quad \mathrm{AUC}=0.630 ; \quad p=0.30, \quad 95 \%$ $\mathrm{CI}=0.521,0.751, \mathrm{AUC}=0.636)$ (see Figure 4).

\section{Discussion}

Stargardt disease is the most common macular dystrophy and no treatments are effectively used in the clinical practice to interrupt the progression of the disease.

The main result of our study was that photo-biomodulation leads to significant improvements in BCVA, PERG amplitude, and fixation location 1 year after treatment $(p<0.001)$, indicating that this therapeutic approach could be beneficial in maintaining visual acuity over time, thus reducing the progression of central visual loss. To our knowledge, this is the first prospective study that has evaluated the effects of photobiomodulation in Stargardt disease, considering a follow-up of 1 year. Previously, in a prospective multicentric study including 36 patients, Scalinci et $\mathrm{al}^{18}$ (ARVO, 2015) observed that BCVA, MP-1, and PERG improved 3months after treatment, suggesting that the role of photo-biomodulation could be helpful in preventing visual loss in patients with Stargardt disease. The role of photo-biomodulation in retinal disease is already known and several studies have demonstrated that this therapy can improve visual acuity in patients with dry agerelated maculopathy (AMD): a study from Merry et al, ${ }^{14}$ from 2017, showed that BCVA and contrast sensitivity significantly improved after 3 months of therapy with a multiwavelength diode, whereas Drusen volume and central Drusen thickness 
FIXATION $2^{\circ}$ BASELINE - FIXATION $2^{\circ} 12 \mathrm{M}$

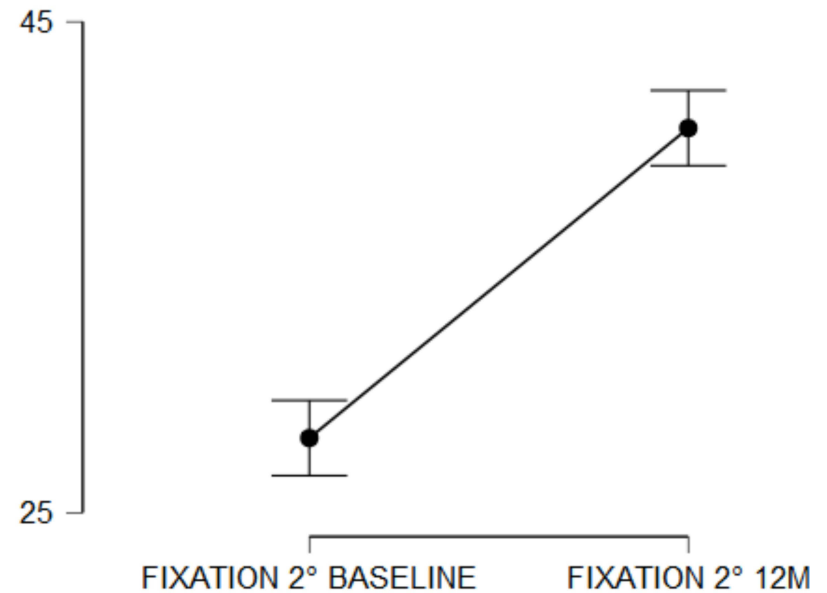

FIXATION $4^{\circ}$ BASELINE - FIXATION $4^{\circ} 12 \mathrm{M}$

76

62

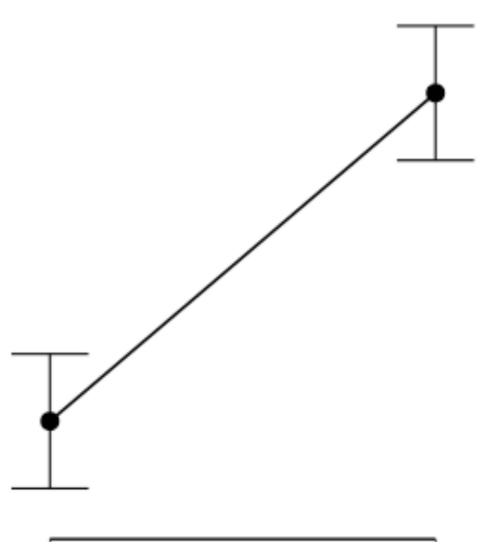

FIXATION $4^{\circ}$ BASELINE

FIXATION $4^{\circ} 12 \mathrm{M}$

Figure 3 The graph shows improvements in fixation tested with MP-I at the baseline and after 12 months of photo-biomodulation. Fixation $2^{\circ}=$ median preferred retinal loci (PRL) within an area of $2^{\circ}$ of diameter; Fixation $4^{\circ}=$ median preferred retinal loci (PRL) within an area of $4^{\circ}$ of diameter.

decreased; moreover, Ivandic et al, ${ }^{13}$ observed that a low level laser therapy of $780 \mathrm{~nm}$ in patients with wet and dry AMD significantly improved visual acuity, compared to a control group.

Also, we observed that PERG N35-P50 amplitude improved rapidly in the first 3 months, whereas after 3 months the curve flattened and the improvement was less consistent (see Figure 1). These findings suggest that photo-biomodulation is more effective in improving PERG amplitude in the first 3 months of treatment, whereas after 3 months its effect decreases.

Another finding in our study was that improvements of BCVA at 12 months were correlated with improvements in
PERG N35-P50 amplitude at 3 and 6 months (respectively, $p=0.042$ and $p=0.025$ ), whereas BCVA improvement was not correlated with improvement of PERG amplitude at 1 month $(\rho=0.103, p=0.332)$. Moreover, we observed that patients with an improvement of more than 1.205 PERG amplitude at 3 months and more than 1.29 at 6 months compared to baseline tended to present an improvement of more than 0.2 in BCVA at 12 months (respectively, $p=0.28$; $p=0.30$ ). These findings suggest that improvements in PERG analysis at 3 and 6 months can estimate the improvement of BCVA and predict visual outcome 1 year after treatment.

Unexpectedly, BCVA improvements were not correlated with improvements of PERG and fixation tested with MP-1 at 12 months. This is a major limitation in our results and we believe that further studies should investigate this point. Moreover, further studies should analyze which parameters might predict clinical improvement in patients treated with photo-biomodulation, in order to clarify in which group of patients this therapeutic approach could be reasonably suggested in clinical practice.

A limitation of the study was the relatively low number of patients recruited. Secondly, patients were not stratified for different ages and onset of the disease. Moreover, photo-biomodulation was performed 5 days a week for 12 months, meaning that the compliance of patients in following the treatment could be low when this therapeutic approach is applied in clinical practice. Also, OCT measures were not recorded and retinal structure was not assessed over time in order to estimate the possible effects of photo-biomodulation. Further prospective studies should investigate the effect of photo-biomodulation over a longer follow-up, also including quantitative structural data of the retina, in order to understand if the protective role of low laser therapy could be maintained over years.

\section{Conclusion}

In our study, we observed that BCVA, PERG N35-P50 and fixation tested with MP-1 significantly improved 1 year after photo-biomodulation. Moreover, improvement of PERG N35-P50 amplitude at 3 and 6 months was correlated to BCVA improvement at 1 year, suggesting that PERG could play an important role in predicting visual outcome 1 year after photo-biomodulation. However, a larger sample of patients, a longer follow-up, and quantitative data of the retina are needed in order to confirm our findings. 


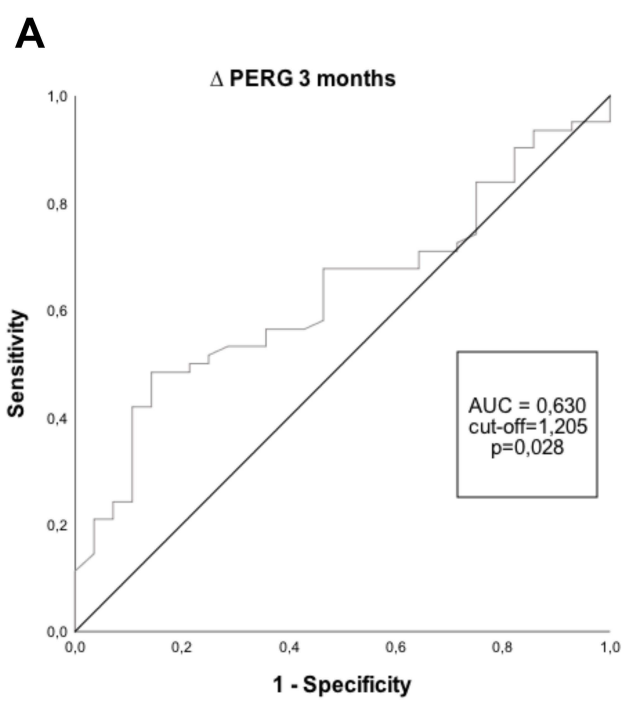

\section{B}

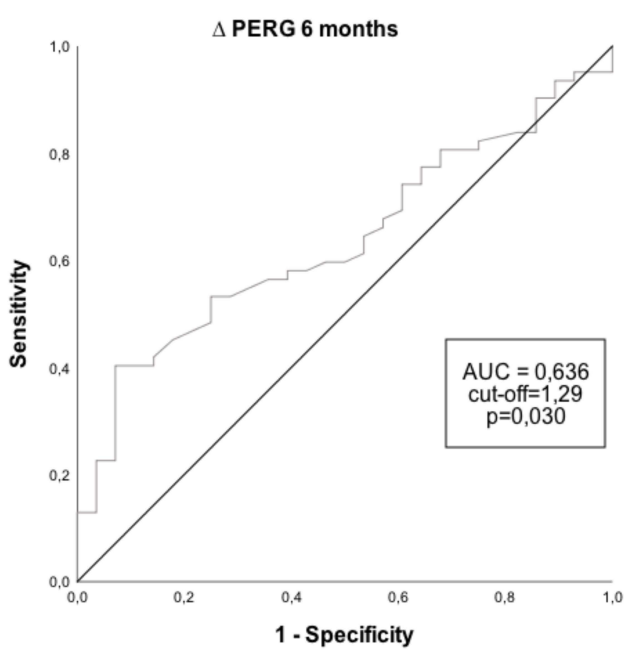

Figure 4 Receiving operating characteristic (ROC) curves were conducted to test if improvements of PERG at I, 3, and 6 months were correlated with an improvement in BCVA. Cut-off 0.2 LogMar was determined after study enrollment in order to evaluate the correlation between PERG improvement and BCVA improvement. Cut-off values were identified with best Youden's Index (Sensitivity + Specificity - I) and tested with Chi-square ROC curve for improvement of PERG at I months. This is not shown as it was not correlated with improvement of BCVA. (A) Patients with an improvement of $>1.205$ at 3 months tend to present an improvement of BVCA $>0.2$ at I year. (B) Patients with an improvement of $>1.29$ at 6 months tend to present an improvement of BVCA $>0.2$ at I year.

Abbreviation: AUC, Area Under the Curve.

\section{Disclosure}

The authors report no conflicts of interest in this work.

\section{References}

1. Michaelides M, Hunt D, Moore A. The genetics of inherited macular dystrophies. J Med Genet. 2003;40(9):641-650. doi:10.1136/ jmg.40.9.641

2. Spiteri Cornish K, Ho J, Downes S, Scott NW, Bainbridge J, Lois N. The epidemiology of stargardt disease in the United Kingdom. Ophthalmol Retina. 2017;1(6):508-513. doi:10.1016/j.oret.2017.03.001

3. Lewis RA, Shroyer NF, Singh N, et al. Genotype/Phenotype analysis of a photoreceptor-specific ATP-binding cassette transporter gene, ABCR, in Stargardt disease. Am J Hum Genet. 1999;64 (2):422-434. doi:10.1086/302251

4. Kniazeva MF, Chiang MF, Cutting GR, Zack DJ, Han M, Zhang K. Clinical and genetic studies of an autosomal dominant cone-rod dystrophy with features of Stargardt disease. Ophthalmic Genet. 1999;20(2):71-81. doi:10.1076/opge.20.2.71.2287

5. Michaelides M, Johnson S, Poulson A, et al. An autosomal dominant bull's-eye macular dystrophy (MCDR2) that maps to the short arm of chromosome 4. Invest Ophthalmol Vis Sci. 2003;44(4):1657-1662. doi:10.1167/iovs.02-0941

6. Zhao PY, Abalem MF, Nadelman D, et al. Peripheral pigmented retinal lesions in stargardt disease. Am $J$ Ophthalmol. 2018;188:104-110. doi:10.1016/j.ajo.2017.12.011

7. Fishman GA. Fundus flavimaculatus. A clinical classification. Arch Ophthalmol Chic Ill. 1976;94(12):2061-2067. doi:10.1001/ archopht.1976.03910040721003

8. Lu LJ, Liu J, Adelman RA. Novel therapeutics for Stargardt disease. Graefes Arch Clin Exp Ophthalmol. 2017;255(6):1057-1062. doi:10.1007/s00417-017-3619-8

9. Karu T. Photobiology of low-power laser effects. Health Phys. 1989;56(5):691-704. doi:10.1097/00004032-198905000-00015

10. Karu TI. Multiple roles of cytochrome c oxidase in mammalian cells under action of red and IR-A radiation. IUBMB Life. 2010;62 (8):607-610. doi:10.1002/iub.359
11. Karu T. Mitochondrial mechanisms of photobiomodulation in context of new data about multiple roles of ATP. Photomed Laser Surg. 2010;28(2):159-160. doi:10.1089/pho.2010.2789

12. Milone FF, Bolner A, Nordera GP, Scalinci SZ. Pulsed led's light at $650 \mathrm{~nm}$ promote and at $470 \mathrm{~nm}$ suppress melatonin's secretion. Neurosci Med. 2015;6(1):35-41. doi:10.4236/nm.2015.61006

13. Ivandic BT, Ivandic T. Low-level laser therapy improves vision in patients with age-related macular degeneration. Photomed Laser Surg. 2008;26(3):241-245. doi:10.1089/pho.2007.2132

14. Merry GF, Munk MR, Dotson RS, Walker MG, Devenyi RG. Photobiomodulation reduces drusen volume and improves visual acuity and contrast sensitivity in dry age-related macular degeneration. Acta Ophthalmol. 2017;95(4):e270-e277. doi:10.1111/ aos. 13354

15. Markowitz SN, Devenyi RG, Munk MR, et al. A double-masked, randomized, sham-controlled, single-center study with photobiomodulation for the treatment of dry age-related macular degeneration. Retina Phila Pa. 2020;40(8):1471-1482. doi:10.1097/IAE.000 0000000002632

16. Ivandic BT, Ivandic T. Low-level laser therapy improves visual acuity in adolescent and adult patients with amblyopia. Photomed Laser Surg. 2012;30(3):167-171. doi:10.1089/pho.2011.3089

17. Ivandic BT, Ivandic T. Low-level laser therapy improves vision in a patient with retinitis pigmentosa. Photomed Laser Surg. 2014;32 (3):181-184. doi:10.1089/pho.2013.3535

18. Scalinci SZ, Milone F, Magnifico M, et al. Photobiomodulation (phototherapy) of retinal tissue in Stargardt disease. Invest Ophthalmol Vis Sci. 2015;56(7):5668.

19. Holder GE. Pattern Electroretinography (PERG) and an integrated approach to visual pathway diagnosis. Prog Retin Eye Res. 2001;20 (4):531-561. doi:10.1016/S1350-9462(00)00030-6

20. Schönbach EM, Wolfson Y, Strauss RW, et al. Macular sensitivity measured with microperimetry in stargardt disease in the progression of atrophy secondary to stargardt disease (ProgStar) Study: report No. 7. JAMA Ophthalmol. 2017;135(7):696-703. doi:10.1001/jamaophth almol.2017.1162 


\section{Publish your work in this journal}

Clinical Ophthalmology is an international, peer-reviewed journal covering all subspecialties within ophthalmology. Key topics include: Optometry; Visual science; Pharmacology and drug therapy in eye diseases; Basic Sciences; Primary and Secondary eye care; Patient Safety and Quality of Care Improvements. This journal is indexed on PubMed

Submit your manuscript here: https://www.dovepress.com/clinical-ophthalmology-journal
Central and CAS, and is the official journal of The Society of Clinical Ophthalmology (SCO). The manuscript management system is completely online and includes a very quick and fair peer-review system, which is all easy to use. Visit http://www.dovepress.com/ testimonials.php to read real quotes from published authors. 